\title{
STANHOPEINAE MESOAMERICANAE IV: LAS CORYANTHES DE CHARLES W. POWELL
}

\author{
Günter Gerlach ${ }^{1} \&$ Gustavo A. Romero-GonzÁlez ${ }^{2}$ \\ ${ }^{1}$ Botanischer Garten München-Nymphenburg, Menzinger Str. 65, D-80638 München, Germany; \\ gerlach@extern.lrz-muenchen.de \\ ${ }^{2}$ Orchid Herbarium of Oakes Ames, Harvard University Herbaria, 22 Divinity Avenue, Cambridge, \\ Massachusetts 02138-2020, U.S.A.; romero@oeb.harvard.edu
}

\begin{abstract}
The descriptions of Coryanthes hunteriana and Coryanthes powellii described by Schlechter are critically compared. We conclude that the same species was described twice. Because of the loss of the holotypes, the specimens collected by Powell with the same collection number at AMES, K and MO are discussed in detail.
\end{abstract}

RESUMEN: Se comparan críticamente las descripciones de Coryanthes hunteriana y Coryanthes powellii ambas descritas por Schlechter. Concluimos que la misma especie se describió dos veces. Como los holotipos se perdieron, se discuten en detalle las muestras existentes del colector Powell en los herbarios AMES, K y MO con el mismo número de colección de los tipos.

Zusammenfassung: Die Beschreibungen von Coryanthes hunteriana und Coryanthes powellii, beide beschrieben von Schlechter, werden kritisch verglichen. Wir kamen zu dem Schluß, daß ein und dieselbe Art zweimal beschrieben wurde. Wegen dem Verlust der Holotypen werden die existierenden Herbarbelege des Sammlers Powell in den Herbarien AMES, K und MO mit denselben Sammelnummern der Typen eingehend diskutiert.

Key words: Orchidaceae, Stanhopeinae, Coryanthes, Powell, Schlechter, Panamá, Costa Rica

La morfología de las flores del género Coryanthes Hook. es sumamente complicada, y casi imposible de describir con palabras. Cada lector puede hacer interpretaciones diferentes de las descripciones y por consiguiente es de gran importancia acompañarlas de un dibujo y, de ser posible, de fotografías mostrando las flores desde diferentes ángulos.

De las dos especies descritas por Rudolf Schlechter de Panamá ( $C$. hunteriana y $C$. powellii) no existen dibujos, pero sí algunas fotografías las cuales, por cierto, al parecer no estuvieron al alcance del autor (al menos él no las mencionó en sus descripciones). En la época de Charles W. Powell (1854 - 1927), quien colectó y cultivó orquídeas principalmente de Panamá desde principios de siglo pasado hasta su muerte, el proceso de tomar e imprimir fotografías de plantas era difícil y relativamente costoso, y por estas razones hay pocas y su calidad no es buena.

Aquí debemos aclarar que el sistema de numeración de muestras empleado por Powell no se ajusta al que está en uso hoy en día: sus números agrupaban especimenes que de acuerdo a su criterio representaban la misma especie, irrespectivamente de donde y cuando hubieran sido colectados y preservados (mientras que ahora numeramos las colecciones de herbario siguiendo el criterio de McNeill et al., 2006: 8, pié de página 1, “... a single gathering of a single species... made by a collector at one time"). En este artículo precisamente tratamos de elucidar las diferentes especies de Coryanthes que Powell distribuyó bajo un mismo número de colección. En algunos casos se puede descartar que algunas de estas muestras puedan ser tipos, porque la fecha que aparece en los rótulos es diferente a la citada en el protólogo. El caso de las muestras sin fecha es ambiguo, aunque en el pasado se han propuesto como lectotipos (Christenson 1991). Aquí continuamos asumiendo que los especímenes sin fecha son tipos, 


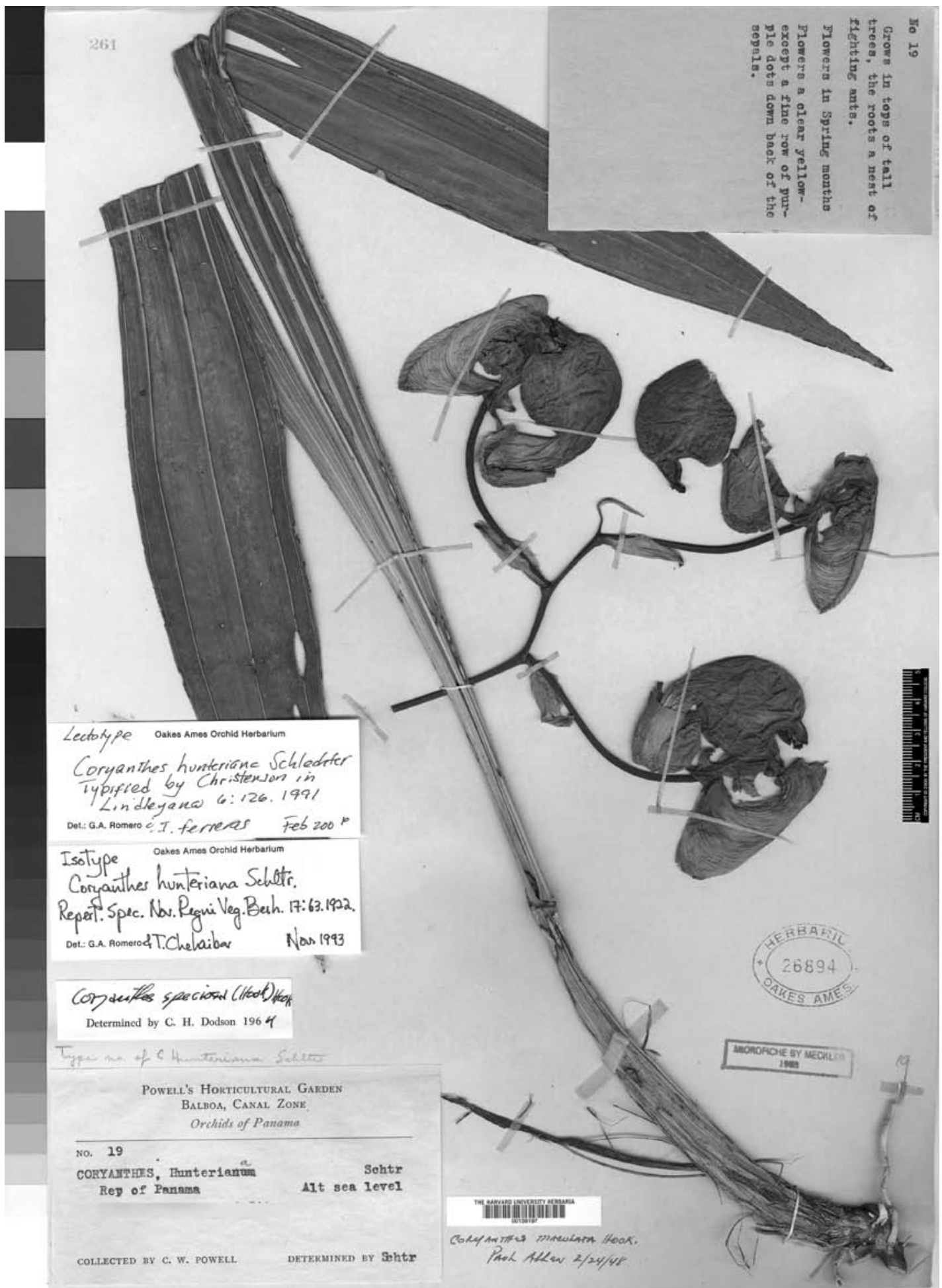

FIGURA 1. Lectotipo de Coryanthes hunteriana Schltr., AMES (26894). 
Tabla 1. Comparación de las descripciones originales en latín de Coryanthes hunteriana Schltr. y C. powellii Schltr.

\begin{tabular}{|c|c|}
\hline Coryanthes hunteriana & Coryanthes powellii \\
\hline $\begin{array}{l}\text { sépalos: } \\
\text { "sepalo intermedio plus minusve crispato, ovato- } \\
\text { suborbiculari, apiculato, vix } 3 \mathrm{~cm} \text { longo et lato, } \\
\text { lateralibus oblique dolabriformibus, a basi usque in } \\
\text { apicem } 6,5 \mathrm{~cm} \text { longis, supra basin margine anteriore } \\
\text { sublobato-dilatatis;" }\end{array}$ & $\begin{array}{l}\text { sépalos: } \\
\text { "sepalo intermedio orbiculari, obtuso, c. } 2,5 \mathrm{~cm} \text { longo } \\
\text { et lato, lateralibus dolabriformibus acuminatis, antice } \\
\text { basin versus sublobato-dilatatis, e basi usque apicem } \\
\text { c. } 7 \mathrm{~cm} \text { longis;" }\end{array}$ \\
\hline $\begin{array}{l}\text { pétalos: } \\
\text { "petalis oblique lanceolato ligulatis, margine crispatis, } \\
\text { c. } 3,5 \mathrm{~cm} \text { longis;" }\end{array}$ & $\begin{array}{l}\text { pétalos: } \\
\text { "petalis oblique ligulatis, obtusiusculis, undulatis } 3,5 \mathrm{~cm} \\
\text { longis;" }\end{array}$ \\
\hline $\begin{array}{l}\text { hipoquilo: } \\
\text { "labelli hipochilio ovatli-cucullato, apice subacuto, } \\
\text { dorso glabro, latere utrinque striguloso-tomentello, 2,5 } \\
\text { cm longo, medio fere } 1,8 \mathrm{~cm} \text { alto," }\end{array}$ & $\begin{array}{l}\text { hipoquilo: } \\
\text { "labelli ungue } 1,7 \mathrm{~cm} \text { longo, hypochilio cucullato, obtuse } \\
\text { acuminato, dorso glabrato, lateribus linea strigillosa } \\
\text { ornato, } 2,7 \mathrm{~cm} \text { longo, medio } 1,8 \mathrm{~cm} \text { alto," }\end{array}$ \\
\hline $\begin{array}{l}\text { mesoquilo: } \\
\text { "mesochilio canaliculato, nudo, hypochilium, paululo } \\
\text { tantum superante," }\end{array}$ & $\begin{array}{l}\text { mesoquilo: } \\
\text { "mesochilio canaliculato, nudo, hypochilium, paulo sed } \\
\text { manifeste superante," }\end{array}$ \\
\hline $\begin{array}{l}\text { epiquilo: } \\
\text { "epichilio generis cucullato, c. } 4 \mathrm{~cm} \text { longo, lobis } \\
\text { lateralibus amplis semiovato-triangularis, obtusis, } \\
\text { antice truncatis, intermedio multo minore semiovalis, } \\
\text { trilobulata" }\end{array}$ & $\begin{array}{l}\text { epiquilo: } \\
\text { "epichilio ovali-cucullato, c. } 4 \mathrm{~cm} \text { longo, lobis } \\
\text { lateralibus amplis semiovatis, antice valde obtusis, } \\
\text { intermedio multo minore oblongo-triangulo trilobulato," }\end{array}$ \\
\hline
\end{tabular}

aunque siempre podrán ser cuestionados (K. Gandhi, comunicación personal, 2008).

De las Coryanthes de Powell existen cuatro fotografías. Todas estas fotografías están montadas en muestras con su número 19. De éstas, una representa C. misasii G. A. Romero \& G. Gerlach, dos representan C. panamensis G. Gerlach y la cuarta tal vez se pueda asignar a una de las dos especies descritas por Schlechter (C. hunteriana, C. powellii).

Las muestras tipos de Schlechter, depositadas en el Herbario de Berlin, fueron destruidas durante la Segunda Guerra Mundial (Merrill, 1943; Ames, 1944). Aunque Schlechter elaboró bosquejos de la mayoría de sus especies y envió copias a su amigo y colega Oakes Ames (Universidad de Harvard, E.E.U.U.; Ames, 1944), no se conocen dibujos de las Coryanthes de Powell. De manera que solamente nos pueden ayudar a elucidar sus dos especies panameñas de Coryanthes las muestras de Powell hoy en día presentes en el Herbario de Orquídeas de los Jardines Reales de Kew (K), en el Herbario de Orquídeas de Oakes Ames en la Universidad de Harvard (AMES) y en el Herbario de Jardín Botánico de Missouri (MO).

En estos tres herbarios hemos localizado siete muestras que llevan el número 19 de Powell, las que, de acuerdo al criterio de Powell y a la descripción de Schlechter, deben pertenecer todas a Coryanthes hunteriana. Además, hay dos muestras de herbario con el número 156 de Powell que, siguiendo de nuevo a Powell y a Schlechter, serían asignadas a $C$. powellii. Christenson (1991) lectotipificó las muestras destruidas en Berlin y aclaró que hubo un error en la cita del número del tipo de esta última especie (Schlechter, por error propio o por un error tipográfico, citó el número 158 de Powell en el protólogo aunque no hay duda que debió haber citado el número 156).

Enseguida examinamos una lista de las 


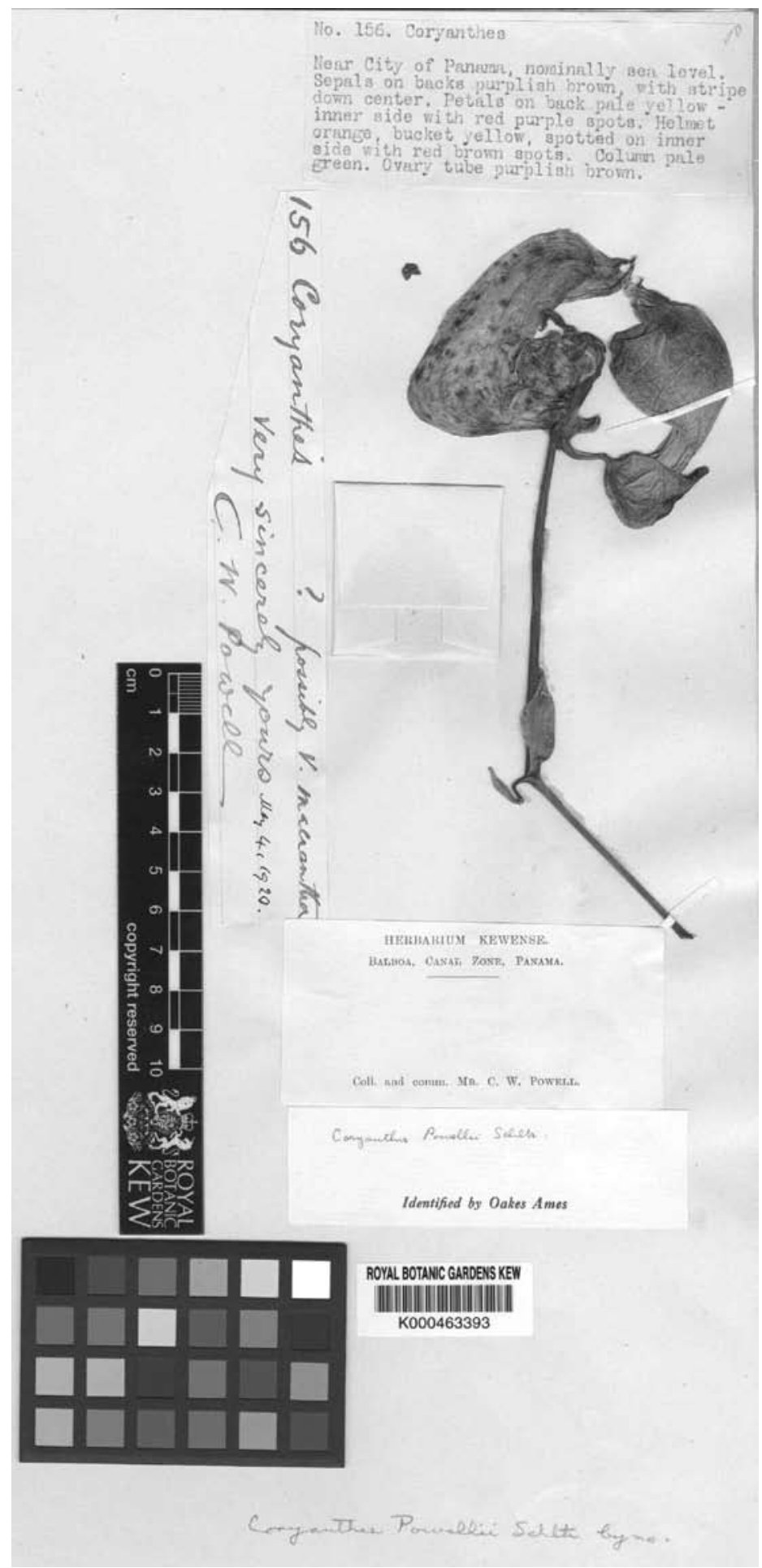

FIgura 2. Lectotipo de Coryanthes powellii Schltr., K. Reproducido con gentil permiso del Board of Trustees of Royal Botanic Gardens, Kew. 


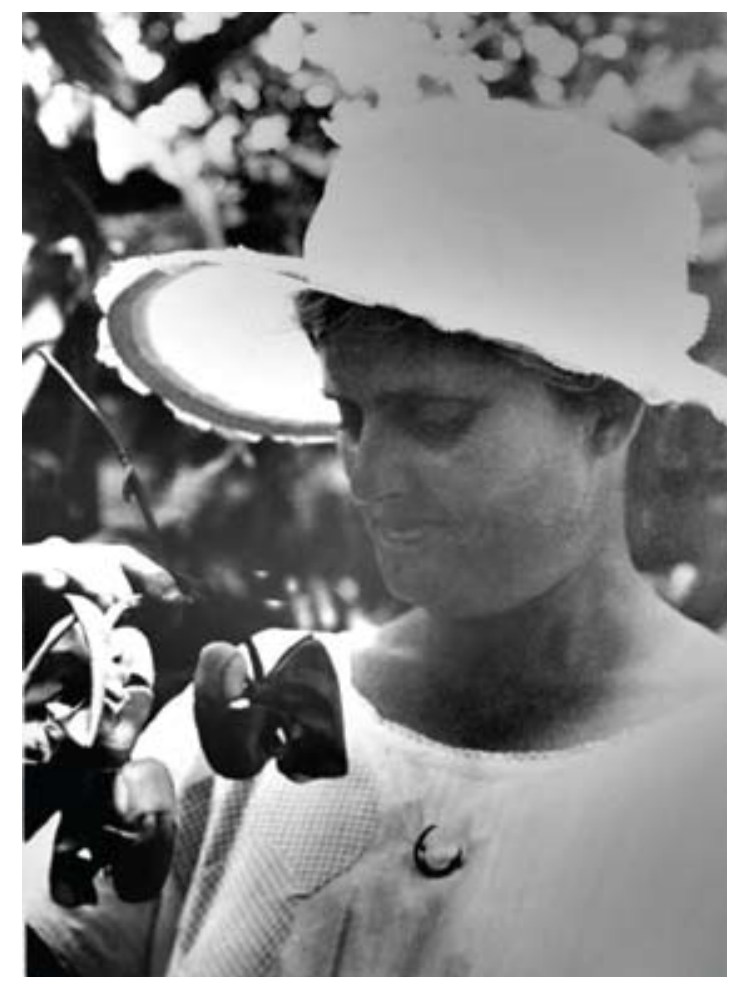

Figura 3. Hija de Charles W. Powell (originalmente montada en la muestra AMES 31853) con Coryanthes panamensis.

muestras existentes de Coryanthes de Powell con algunos comentarios.

Bajo Powell 19 - todo este material se ha identificado como Coryanthes hunteriana Schltr.

1) AMES 26894: Lectotypus (Fig. 1), no contradice el protólogo, sin fecha; labelo $5,9 \mathrm{~cm}$ alto;

2) AMES 25007: Isolectotypus, no contradice el protólogo, sin fecha; labelo $5,7 \mathrm{~cm}$ alto;

3) AMES 25008: la planta, por falta de flores, no es determinable, la muestra incluye una foto de $C$. misasii, tiene fecha del 5 de mayo de 1923;

4) $\mathrm{K}$ (sin número de herbario): Isolectotypus, cinco flores de C. panamensis, dos fotografías (la del habito no se puede determinar, la de la flor la identificamos como C. panamensis), tiene fecha del 15 de noviembre de 1918; labelo $6,3 \mathrm{~cm}$ alto;

5) MO 955890: no contradice el protólogo, sin fecha; labelo 5,6 cm alto;

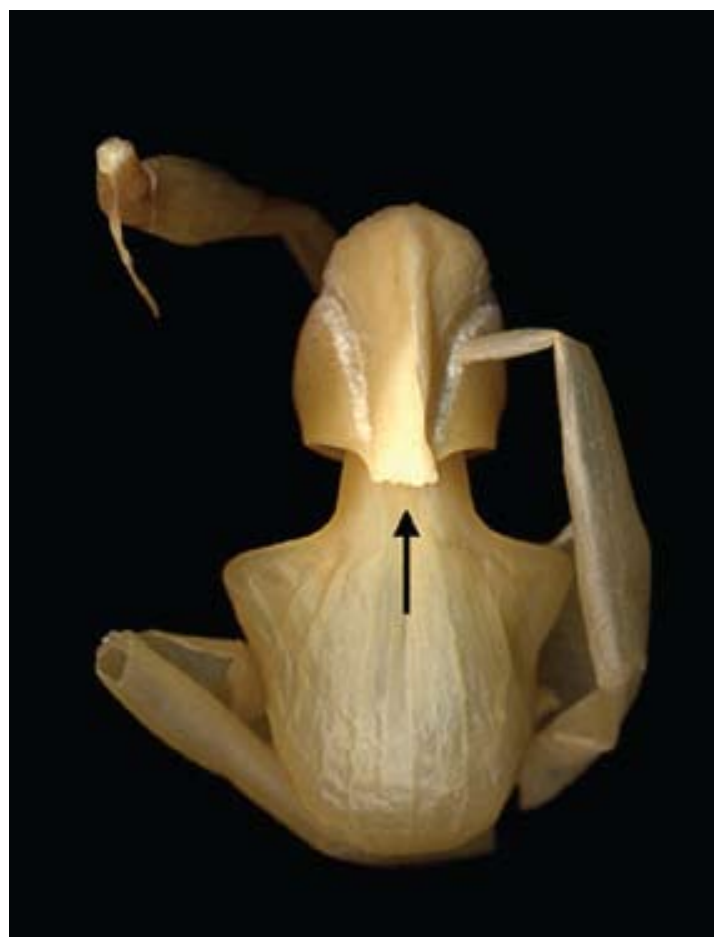

Figura 4. Coryanthes hunteriana Schltr., Powell 156 (conservado en líquido en AMES); la flecha muestra el hipoquilo alargado y truncado, el carácter más distintivo de la especie.

6) AMES (sin número de herbario) Powell 3546 y Powell 19 (ambas anotaciones sobre rótulo), con fecha de abril de 1924;

7) AMES 31853: Powell 19 y Oakes Ames 3519; una flor y una foto de la hija de Powell (Fig. 3) con una flor de C. panamensis, con fecha de marzo de 1924.

Bajo Powell 156 - todo este material se ha identificado como Coryanthes powellii Schltr.

1) K (sin número de herbario): Lectotypus (Fig. 2), una una flor montada al revés, la flor mas delgada que la de $C$. hunteriana, tiene fecha del 4 de mayo de 1920, labelo 5,9 cm alto;

2) AMES 26893: se parece a AMES 26894 y todavía más a AMES 25007 (C. hunteriana), sin fecha; labelo $5,8 \mathrm{~cm}$ alto.

En este material de herbario se encuentran dos especies de Coryanthes adicionales que fueron descritas 


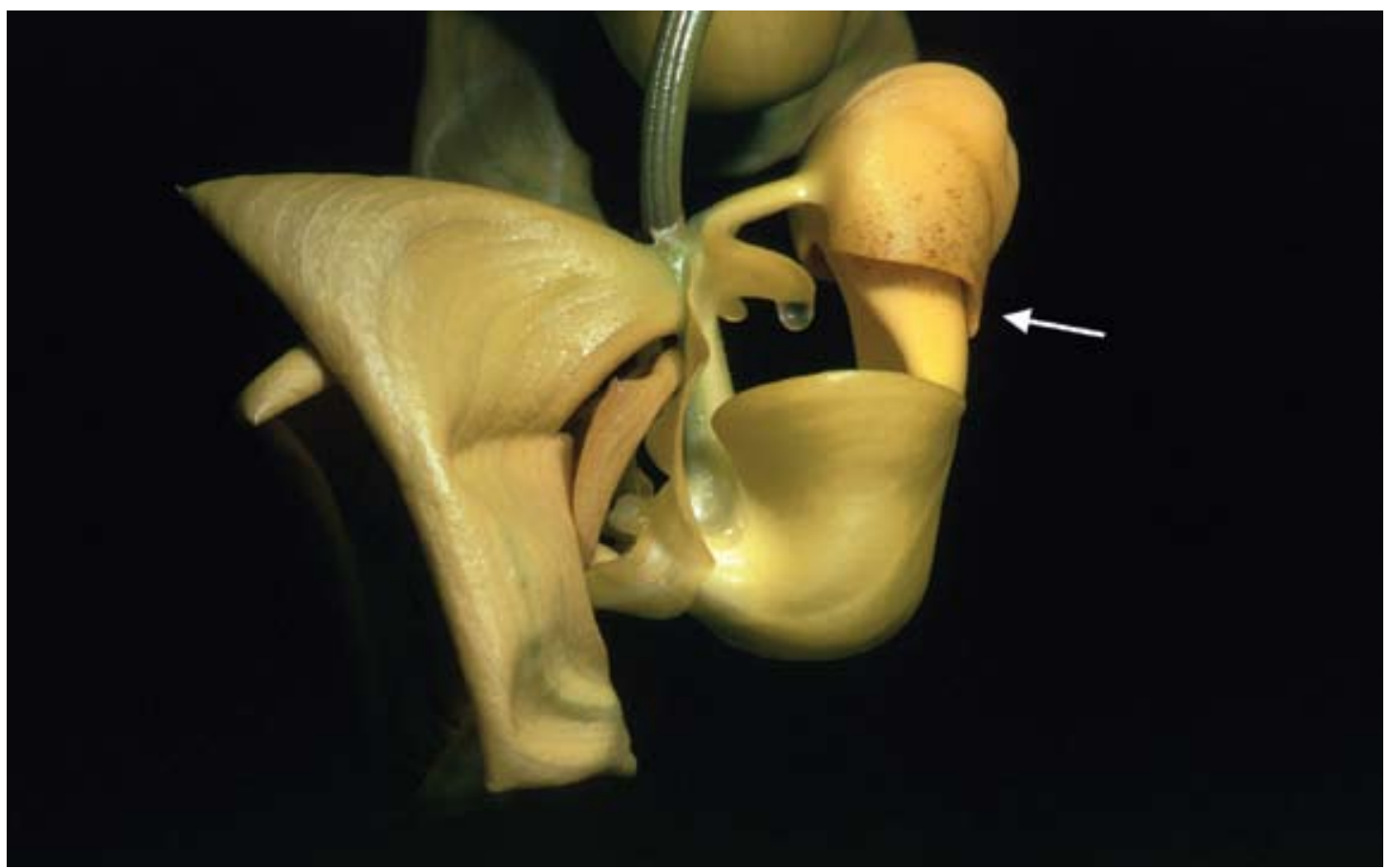

Figura 5. Coryanthes hunteriana Schltr., Panamá, Prov. Panamá, cerca de Arraiján, 02/2948, vista lateral; la flecha muestra el hipoquilo alargado, el carácter más distintivo de la especie. Foto G. Gerlach.

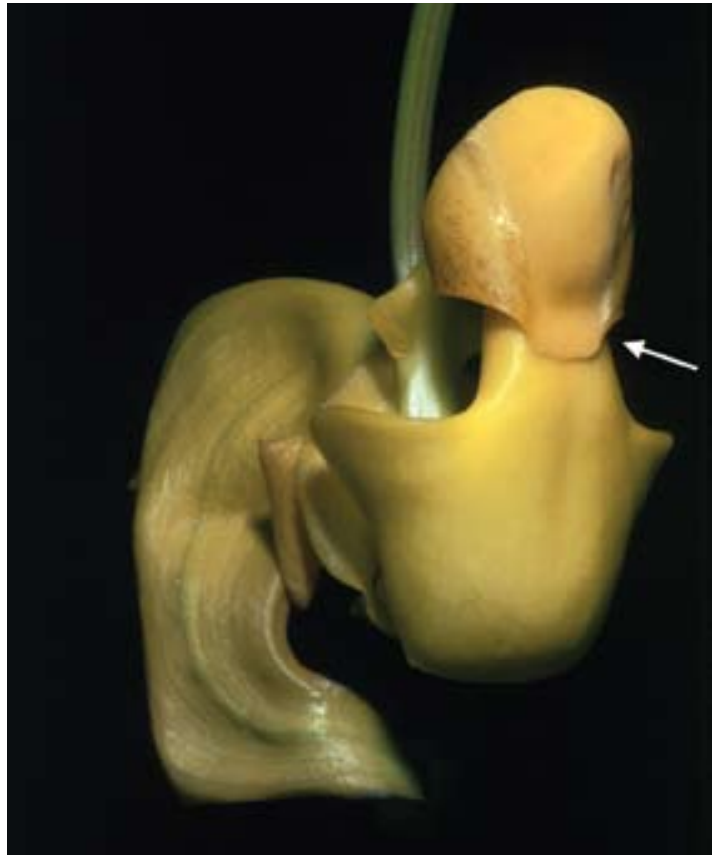

Figura 6. Coryanthes hunteriana Schltr., Panamá, Prov. Panamá, cerca de Arraiján, 02/2948, vista dorsal; la flecha muestra el hipoquilo alargado y truncado, el carácter más distintivo de la especie. Foto G. Gerlach.

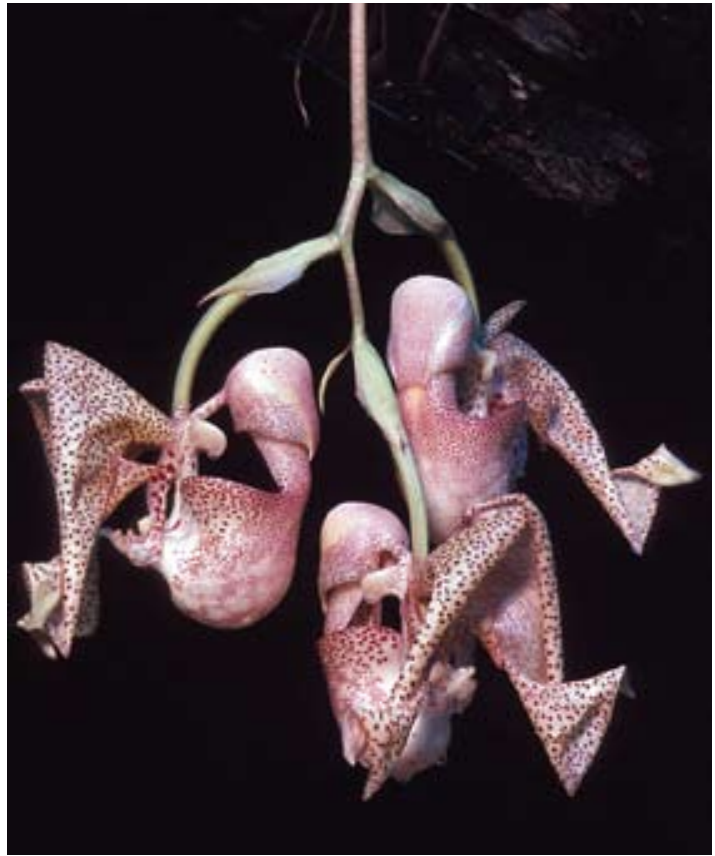

Figura 7. Coryanthes hunteriana Schltr., Costa Rica, Prov. Limón, Río Pacuare, O-18363 (forma rosada que representando el color de tipo de C. horichiana). Foto G. Gerlach. 


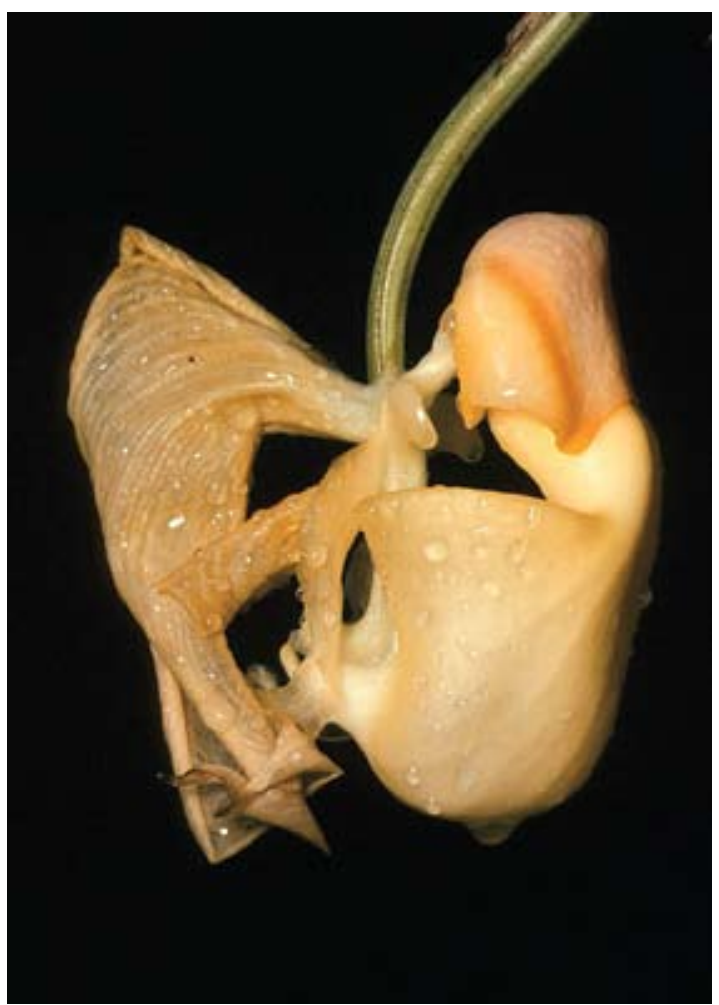

Figura 8. Coryanthes panamensis G. Gerlach, Panamá, Prov. Panamá, Río Piedras (zona del canal) O-18594 (fotografía del material del holotipo), vista lateral. Foto G. Gerlach.

décadas después como C. misasii G.A. Romero \& G. Gerlach (Gerlach \& Romero 1990) y C. panamensis G. Gerlach (Gerlach \& Schill 1993). Comparando las flores de las llamadas $C$. hunteriana y $C$. powellii, no encontramos diferencias que justifiquen el estatus de C. powellii como especie o subespecie. Comparando las descripciones originales (i.e., los protólogos) de las dos especies tampoco se detectan grandes diferencias. Además, aquí enfatizamos que estas descripciones se basan en caracteres que tienen poco peso para distinguir las especies. La tabla 1 contrasta las descripciones originales en latín.

Trabajando con material extenso del género Coryanthes, tanto cultivado como de herbario, hemos determinado que los caracteres que permiten identificar las especies (i.e., los caracteres diagnósticos) no están siempre incluidos en las descripciones. Gerlach (1993) diferenciaba su C. panamensis por el ápice

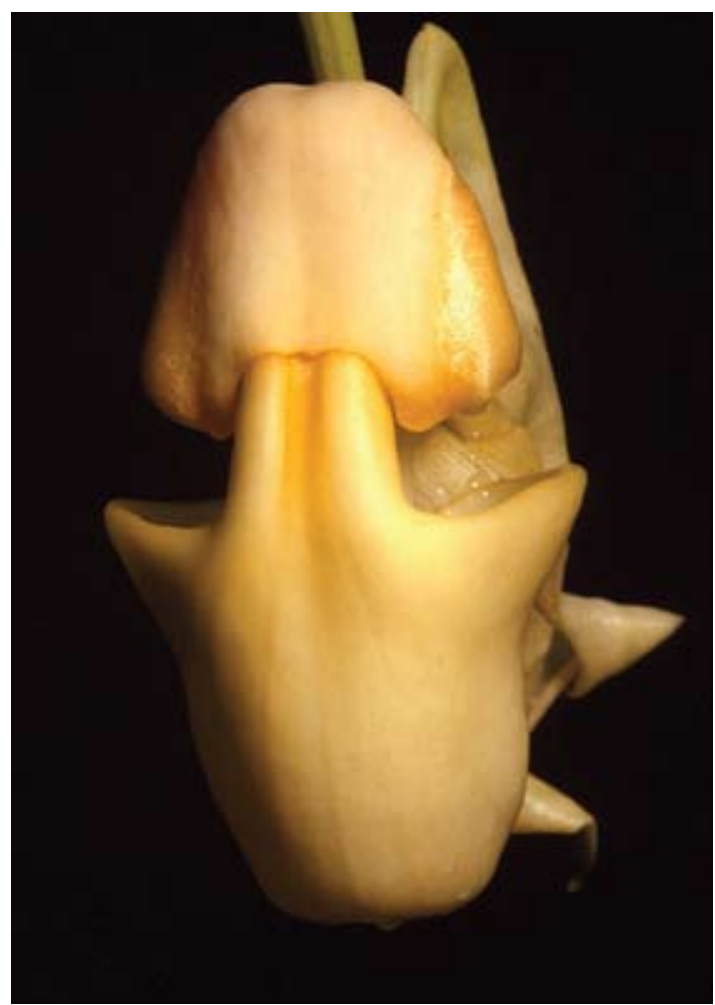

Figura 9. Coryanthes panamensis G. Gerlach, Panamá, Prov. Panamá, Río Piedras (zona del canal) O-18594 (fotografía del material del holotipo), vista dorsal. Foto G. Gerlach.

del hipoquilo profundamente trapeziforme y sinuado (“... Cum apice hypochilii profundo trapeziformesinuato"). En sus descripciones Schlechter mencionó al caracter del hipoquilo: "apice subacuto" en C. hunteriana y "hypochilio cucullato, obtuse acuminato" en C. powellii. En realidad, el hipoquilo se ve subagudo o obtuso acuminado en todas las flores menos en aquellas que nosotros hemos identificado como Coryanthes panamensis y C. misasii. Estas dos últimas especies no presentan el carácter del ápice del hipoquilo mencionado por Schlechter (Fig. $8-12$ ) y por consiguiente no se pueden confundir con sus conceptos de C. hunteriana o $C$. powellii. Comparando todo este material con los números mencionados 19 y 156 de Powell, creemos que Schlechter tuvo en sus manos la misma especie pero en dos oportunidades con diferentes números $\mathrm{y}$, sin darse cuenta, la describió dos veces (un error que este 

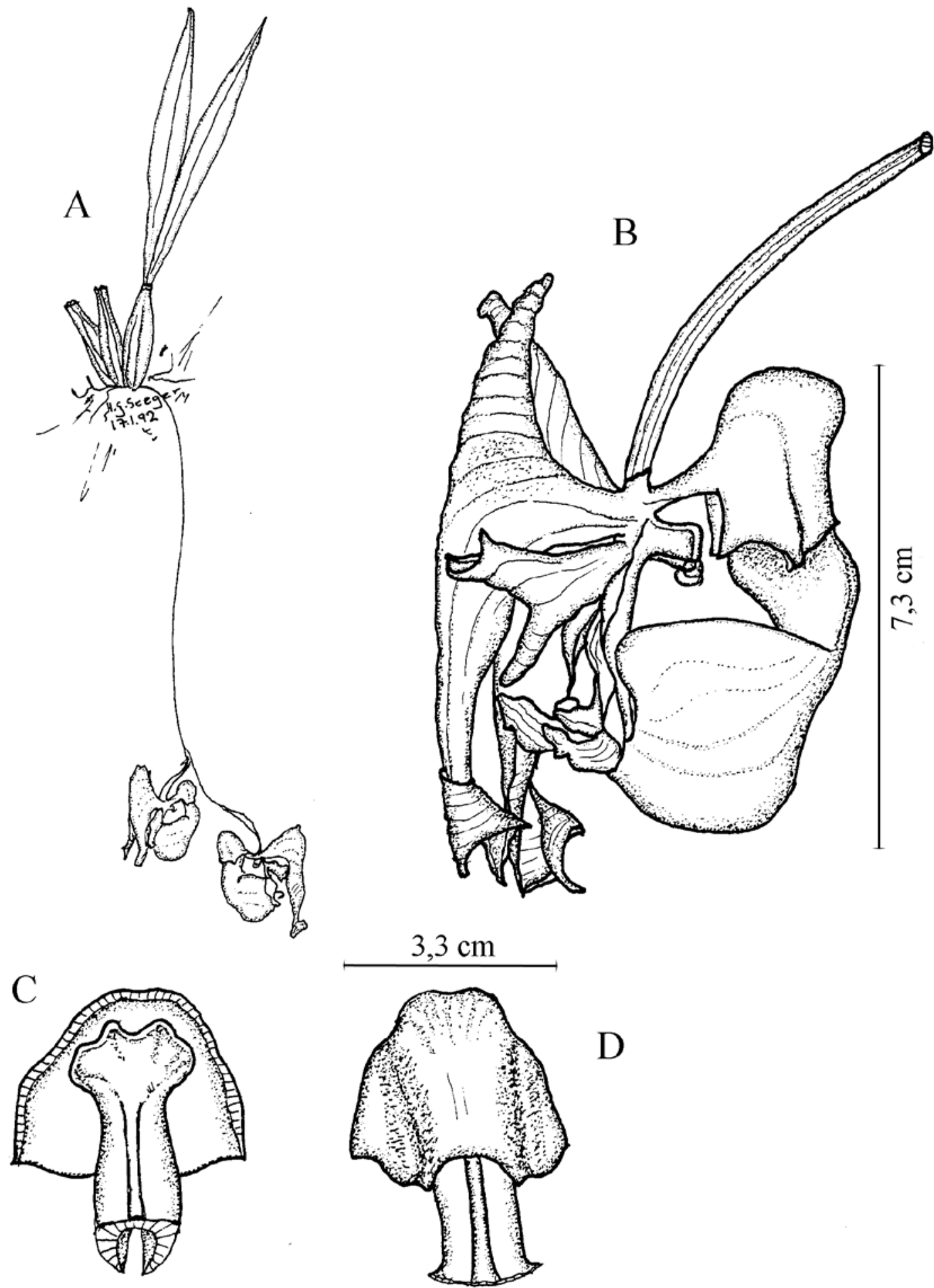

Figura 10. Coryanthes panamensis G. Gerlach. - A. habito, - B. Flor en vista lateral, - C. Osmoforo, partes del hipoquilo removido, - D. Hipoquilo en vista dorsal. Dibujo por H.G. Seeger. 


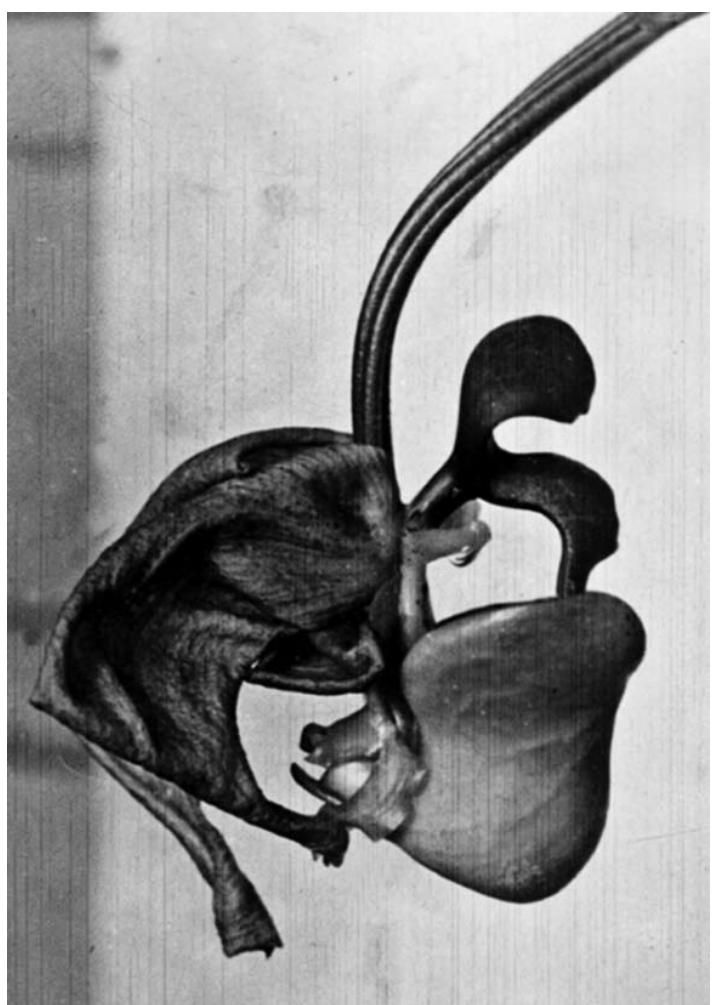

Figura 11. Fotografía de Coryanthes misasii G. A. Romero \& G. Gerlach (originalmente montada en la muestra AMES 25008).

autor cometió en varias oportunidades). Esta hipótesis se apoya en la falta de diferencias significativas en las flores prensadas por Powell con estos números y la falta de diferencias importantes en las descripciones de Schlechter de C. hunteriana y C. powellii (Tabla 1). Basándonos en estos argumentos, referimos a $C$. powellii a la sinonímia de $C$. hunteriana.

Allen (1949) fue el primer botánico en relegar las dos especies de Schlechter a la sinonimía a Coryanthes maculata Hook., aunque también incluyó en su lista de sinónimos a C. albertinae H. Karst. y C. splendens Barb. Rodr. Pensamos que el concepto de Coryanthes de Allen era muy amplio y que incluía muchas especies que ahora son aceptadas separadamente por muchos autores, como C. albertinae, restringida a Venezuela, C. maculata, un elemento típico de la Flora del escudo Guayanés, y $C$. splendens, un sinónimo de C. speciosa (Hook.) Hook., una especie endémica de Brasil.

Debido a la falta de caracteres diagnósticos descritos
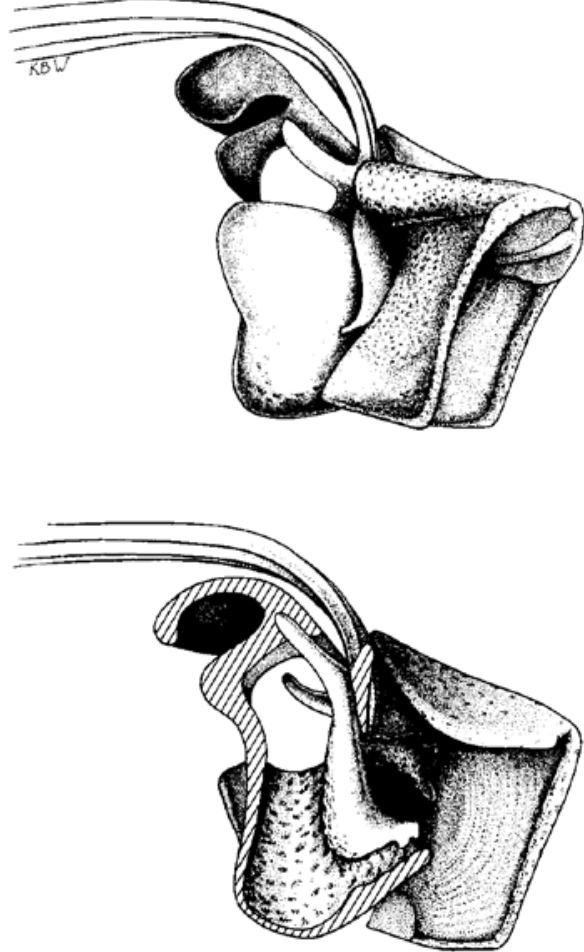

Figura 12. C. misasii G. A. Romero \& G. Gerlach. Dibujo por K. Brown-Wing.

por Schlechter que pudieran servir para identificar Coryanthes hunteriana, aquí complementamos su descripción para que esta especie pueda ser identificada con seguridad en el futuro. Se distingue fácilmente por la forma del ápice del hipoquilo: en vista lateral éste se ve largamente elongado (Fig. 5 y 7) y en vista dorsal truncado o obtuso (Fig. 4 y 6). En la mayoría de las especies de la sección Coryanthes del género Coryanthes (en Mesoamérica no hay reportes de la otra otra sección) el ápice del hipoquilo se ve emarginado y sinuado (por ejemplo en C. panamensis, Fig. 8, 9 y 10) o agudo, pero en ningún caso truncado o obtuso.

La interpretacion de C. hunteriana por Gerlach \& Schill (1993) en su monografía del género Coryanthes estuvo errada: la especie ilustrada con este nombre hoy se asigna a $C$. picturata, la especie más común en Mesoamérica, que se encuentra desde México hasta Panamá. Es bastante variable y tiene formas unicolores de color crema o amarillo pálido igual que formas punteadas o manchadas. La especie que está ilustrada 
en la misma obra con el nombre Coryanthes picturata (Gerlach \& Schill 1993: página 79, figura 10 y página 146 figuras 90 \& 91), tampoco fue correctamente interpretada: ésta fue descrita diez años más tarde como C. kaiseriana G. Gerlach (Gerlach \& Dressler 2003).

Para concluir, hoy en día el conocimiento y los datos que tenemos disponibles de Coryanthes claramente son mucho mejores que en el pasado, debido a una mejor infraestructura de información y a que se han colectado más muestras de las diferentes especies del género. Las especies de Coryanthes descritas por Schlechter de Panamá ya han sido colectadas en Costa Rica, aunque las flores presentan una coloración algo diferente (Fig. 7). Supuestamente por causas de la descripción pobre en caracteres y por falta de ilustraciones, Jenny (1986) la describió como C. horichiana sin darse cuenta que se trataba de la $C$. hunteriana.

Agradecimientos. Agradecemos a los herbarios y los curadores del Royal Botanical Gardens, Kew en Inglaterra (K), y del Jardín Botánico de Missouri, San Louis, Missouri, E.E.U.U. (MO), por permitirnos estudiar sus colecciones y publicar algunas de sus muestras. Los autores agradecen el apoyo financiero de la Sociedad de Orquideología de Arizona (Orchid Society of Arizona, a G.A.R.-G.).
LiterATURA CitADA

Allen, P.H. 1949. Flora of Panama. Part II. Fascicle 4. (Orchidaceae, third part). Ann. Miss. Bot. Gard. 36(1): 66-69.

Ames, O. 1944. Destruction of the Schlechter Herbarium by bombing. Amer. Orchid Soc. Bull. 13: 105-106.

Christenson, E.A. 1991. Mesoamerican orchid studies II: Powell's panamanian orchidaceae. Lindleyana 6(3): 124-132.

Gerlach, G. \& Romero, G.A. 1990. Beiträge zur Gattung Coryanthes Hook. VI: Coryanthes gernotii und Coryanthes misasii zwei neue Orchideen aus den Wäldern Südamerikas. Die Orchidee 42(2): 64-68.

Gerlach, G. \& Schill, R. 1993. Die Gattung Coryanthes Hook. (Orchidaceae); Eine monographische Bearbeitung unter besonderer Berücksichtigung der Blütenduftstoffe. Tropische und subtropische Pflanzenwelt 83: 1-205.

Gerlach, G. \& Dressler, R. 2003. Stanhopeinae Mesoamericanae I. Lankesteriana 8: 23-30.

Jenny, R. 1986. Einige Notizen zur Gattung Coryanthes Hooker und eine neue Art: Coryanthes horichiana. Die Orchidee 37(3): 124-132.

McNeill, J. [Chairman]. 2006. International Code of Botanical Nomenclature (Vienna Code). A. R. G. Gantner Verlag, Ruggell, Liechtenstein.

Merrill, E. D. 1943. Destruction of the Berlin Herbarium. Science 98, No. 2553: 490-491.

Schlechter, R. 1922. Beiträge zur Orchideenkunde von Zentralamerika. I. Orchidaceae Powellianae Panamenses. Repert. Spec. Nov. Regni Veg. Beih. 17: 1-95. 